\title{
Performance standard only half done
}

$t$ is easy to understand why the IACUC would be concerned when observing the Xenopus in an algae-covered tank; after all, most IACUC members are accustomed to seeing furry animals in clean cages as most institutions probably utilize warm-blooded animals more than cold-blooded ones. Yet this scenario is important because Xenopus are probably the most widely used amphibian in animal research today.

The Guide for the Care and Use of Laboratory Animals does not contain many frog-specific recommendations for the care of these animals. This could leave the investigators and the IACUC at a bit of a loss when it comes to evaluating the animals during a semi-annual inspection. But it is important to realize that even

\section{A WORD FROM OLAW}

In response to the issues posed in this scenario, the National Institutes of Health - Office of Laboratory Animal Welfare (NIH-OLAW) provides the following clarification:

In this scenario, IACUC inspectors report concerns to the committee about algae overgrowth in tanks in a satellite Xenopus frog room. Because there are no apparent health problems with the frogs housed in the more natural habitat, the principal investigator is not concerned and does not find it outside the standards of care.

The PHS Policy requires the IACUC, as an agent of the institution, to inspect at least once every six months all of the institution's animal facilities including satellite facilities using the Guide for the Care and Use of Laboratory Animals as a basis for evaluation ${ }^{1}$. The expectations of both the PHS Policy and the Guide is for institutions to establish standards for IACUC and veterinary oversight of all animal species whether housed in central or satellite facilities ${ }^{2-4}$. The Guide states that "establishing standard operating procedures can assist an institution in complying with regulations, policies, and principles as well as with day-to-day operations and management ${ }^{2}$." While it is the IACUC's responsibility to review husbandry standards that directly impact animal welfare, it may engage the expertise of others to assure the standards are appropriate for the species. The IACUC may also allow standards that deviate from commonly acceptable practices though the Guide may have few specific recommendations, many of the general recommendations of the Guide apply. We would argue that the most important recommendation would be the use of performance standards.

The Guide identifies a performance standard as "a standard or guideline that, while describing a desired outcome, provides flexibility in achieving this outcome by granting discretion to those responsible for managing the animal care and use program, the researcher, and the IACUC." "

In this scenario, one could argue that, given a paucity of regulatory standards for the housing of Xenopus, a performance standard should be the guiding principal when the evaluation of the care of Xenopus is performed. It appears that the

if justified for scientific reasons ${ }^{5}$. Likewise, the veterinarian may allow deviations for veterinary care or husbandry reasons. Standard operating procedures or written performance standards are clearly needed for Great Eastern University's frog room that address water turbidity and ensure daily observations, adequate oxygenation, and limited biofilm accumulation while providing a more natural habitat ${ }^{2}$.

\section{Patricia Brown $\bowtie$}

Director, OLAW, OER, OD, NIH, HHS, Bethesda, $M D$, USA.

凶e-mail:brownp@od.nih.gov

Published online: 23 July 2020

https://doi.org/10.1038/s41684-020-0600-7

References

1. Office of Laboratory Animal Welfare, National Institutes of Health. Public Health Service Policy on Humane Care and Use of Laboratory Animals. (US Department of Health and Human Services, Bethesda, Maryland, USA, 2015). https://olaw.nih.gov/ policies-laws/phs-policy.htm

2. Institute for Laboratory Animal Research. Guide for the Care and Use of Laboratory Animals. 8th edn. (National Academies Press, Washington, D.C., 2011).

3. National Institutes of Health. Public Health Service Policy on Humane Care and Use of Laboratory Animals - Frequently Asked Questions. Animal Use and Management, Question F.16. (US Department of Health and Human Services, Bethesda, MD, USA, revised 2017). https://olaw.nih.gov/guidance/faqs $\#$ f16 (accessed June 15, 2020).

4. National Institutes of Health. Public Health Service Policy on Humane Care and Use of Laboratory Animals - Frequently Asked Questions. Institutional Responsibilities, Question G.10. (US Department of Health and Human Services, Bethesda, MD, USA, revised 2017). https://olaw.nih.gov/guidance/faqs\#g10 (accessed June 15, 2020).

5. Brown, Patricia \& Gipson, Chester Lab Animal 41, $41-43$ (2012). implementation of this standard is what has broken down.

The Principle Investigator (PI),

Dr. Eaton, and his staff have been caring for the Xenopus as allowed by the IACUC. The PI is of the understanding that all is fine because the frogs' housing is as 'natural' as possible, it was discussed with animal facility personnel, and there appeared to be no health problems for the animals. The fact that the animals are healthy is of prime importance. The IACUC, however, appears to be unaware of the specifics and therein lies the problem.

The Attending Veterinarian (AV), as opposed to simply "animal facility personnel," should have discussed the husbandry and care of the animals with Eaton to determine what would be appropriate. As part of the IACUC protocol, the PI should provide a Standard Operating Procedure (SOP) of the husbandry schedule, which would include documentation of items such as water changes, water testing, temperature, feeding, etc. In that way, the IACUC would have known that there may be algae in the tanks and that its presence in the tanks does not equal inadequate care of the frogs. The AV could explain the procedures as to how the animals will be maintained by the researchers to the IACUC during the protocol review process. If the AV had little experience with Xenopus, and the IACUC was concerned, they could invite a biologist who has experience with these animals to advise them.

The ultimate indicator of adequate care for this species is going to be the health of the animals. In this scenario, it appears that the animals are healthy so the answer to the question is yes, the IACUC should have some concerns but those concerns should not necessarily be regarding the outcome (as the animals are healthy) but the process used by which that outcome was achieved (husbandry and care procedures).

\section{Alison D. Pohl ${ }^{\bowtie}$ and Ron G. Wallace UConn Health, Farmington, CT, USA.

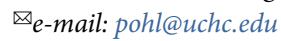

Published online: 23 July 2020

https://doi.org/10.1038/s41684-020-0598-X

\footnotetext{
References

1. Institute for Laboratory Animal Research. Guide for the Care and Use of Laboratory Animals 8th ed. (National Academies Press, Washington, DC, 2011).
} 